\title{
Massive hemoperitoneum for spontaneous rupture of unscarred uterus in the third trimester of pregnancy: A case
} report

Debora D'aniello*, Giusi Santangelo, Valentina Tibaldi, Andrea Giannini, Violante Di Donato, Roberto Brunelli, Innocenza Palaia, Ludovico Muzii, Pierluigi Benedetti Panici and Marco Monti

Department of Maternal and Child Health and Urological Sciences, University of Rome “Sapienza”, Umberto I Hospital, Italy

\begin{abstract}
A spontaneous uterine rupture during the pregnancy is associated with high mortality and morbidity in both the fetus and the mother.

We report the case of a rupture in an unscarred uterus in a gravid woman, $29+6$ weeks of gestation, with history of uterine curettage for previous miscarriage. The maternal and fetal clinical status rapidly deteriorated and required emergency caesarean delivery with hysterectomy. A massive hemoperitoneum was found and the cause of the event was the uterine rupture.

This case shows the importance of good practice and that the survival of the patients depends on the time between rupture and intervention of medical staff. All the previous uterine surgeries report an increase of the risk of placenta percreta at the site of the prior scar.
\end{abstract}

\section{Introduction}

The term 'ruptured uterus' covers a number of conditions which should be classified according to the site of rupture and the presence or absence of a scar from previous caesarean section. The rupture of the pregnant uterus is a serious obstetrical event associated a high perinatal and maternal morbidity including blood transfusion, hysterectomy, neonatal respiratory distress, perinatal asphyxia, urological injury and maternal and/or fetal mortality. It is a rare occurrence (3-5.9 per 10,000 pregnancies) [1-3] and the incidence of the uterine rupture with previous caesarean is $1 \%$, while for the primigravid is an extremely rare condition $(0.012 \%)$ [1]. The total risk of rupture after abdominal myomectomy is $0-4 \%$ and after laparoscopy $0-1 \%$. [4]. It seems that uterine rupture after caesarean section occur more commonly.

The prevalence of the uterine rupture is higher in developing countries than the developed countries cause by worse obstetrical care, multiparity and the high frequency of home deliveries with prolonged labor. Between 3.1 and $12.9 \%$ of uterine ruptures in developed countries occur in an unscarred uterus $[5,6]$.

Rupture during trial of labor, in a patient with a scar is not nearly as serious as rupture occurring outside hospital in a patient with a previously intact uterus. Fetal mortality is significant, however, in both conditions.

We report a case report of a spontaneous uterine rupture at 30 weeks of gestation in a woman who has never had a previous caesarean, but who has had uterine curettage for a previous spontaneous abortion.

\section{Case report}

A 38-year-old woman, para 0/0/1/0, pregnant at 29 weeks and 6 days came to our Department for abdominal pain. She had a past history of first trimester miscarriage. The patient reported a previous hospitalization for uterine contraction and in that situation, she received prophylactic tocolytics and cortisone.

On admission her blood pressure was $100 / 60 \mathrm{mmHg}$, pulse rate was 72 beats $/ \mathrm{min}$, body temperature was $36^{\circ} \mathrm{C}$, and a normal respiratory rate.

The obstetrical examination revealed: posterior cervical position, moderate consistency and the cervical orifice was close. No vaginal bleeding. The fetal heart rate pattern was reassuring, and no uterine contractions were seen. Transabdominal ultrasonography identified a normal amniotic fluid index and confirmed the vertex presentation. Twelve hours after the recovery she reported nausea with an improvement of the pain and the cardiotocography (CTG) showed a reassuring fetal heart rate pattern, no contractions.

Four hours later, during the CTG were observed sinusoid oscillation and variable decelerations (Figure 1) and the patient was immediately carried to the delivery room. After 20 minutes of CTG monitoring the continuous deceleration and the severe sustained abdominal pain, and in particularly the patient's hemoglobin dropped from $10.9 \mathrm{~g} / \mathrm{dl}$ at the time of admission to $7.5 \mathrm{~g} / \mathrm{dl}$ with a blood pressure of $60 / 40 \mathrm{mmHg}$, the gynecologist decided for an emergency caesarean section and the patient was moved to the operating room.

${ }^{*}$ Correspondence to: Debora D'aniello, Department of Maternal and Child Health and Urological Sciences, University of Rome "Sapienza", Umberto I Hospital, Italy, Tel: 39064940550; Fax: 390649972564; E-mail: debora.daniello@ uniromal.it

Key words: uterine rupture, hemoperitoneum, hysterectomy, placenta percreta

Received: September 18, 2020; Accepted: September 25, 2020; Published: September 28, 2020 


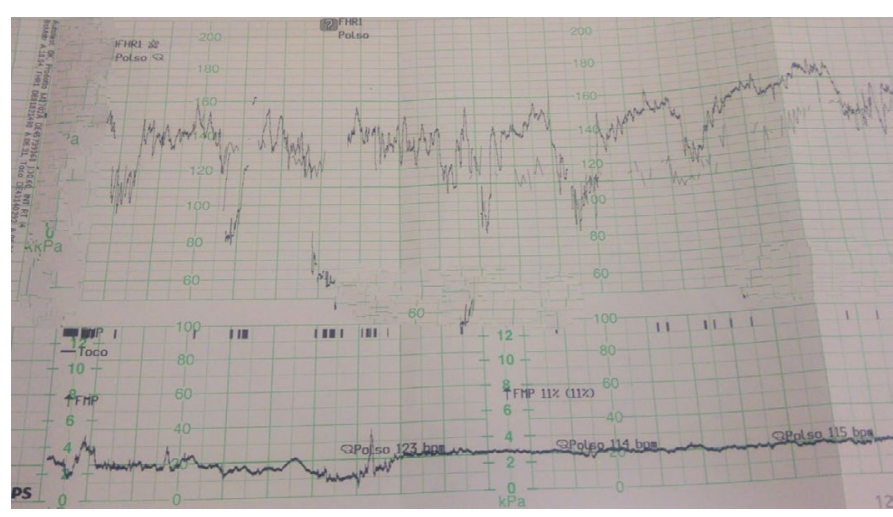

Figure 1. Atypical variable decelerations on cardiotocography

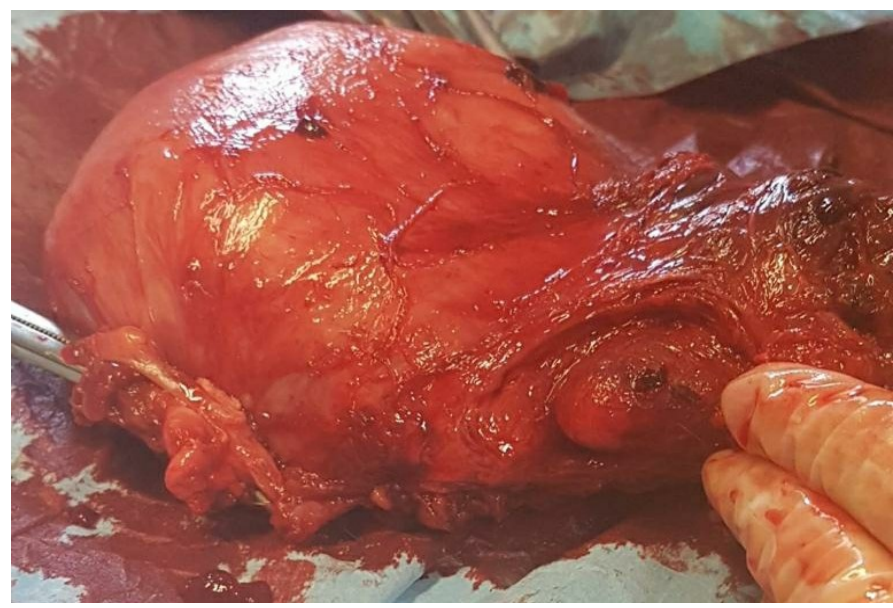

Figure 2. Rupture in the posterior and right lateral wall of the uterus

A massive hemoperitoneum approximately 3-4 liters of blood was found, and a hysterectomy was performed in response to the irreversible damage to the uterus and uncontrolled hemorrhage. A rupture with disruption of all layers measuring $4 \mathrm{~cm}$ was observed in the posterior and right lateral wall of the uterus (Figure 2). The total hysterectomy and peritoneal toileting were performed. The patient had received a transfusion with two units of red blood cells intraoperatively. The birth weight was $1420 \mathrm{~g}$ and Apgar scores were 3 at born, 6 at 1 min and 8 at $10 \mathrm{~min}$, respectively. The patient was then transferred to the intensive care unit where she was followed for 36 hours. Her hemoglobin level in the first post-operative day was $6.3 \mathrm{~g} / \mathrm{dl}$. She received other two units packed of erythrocyte concentrate and one unit of plasma.

The patient remained at the department of Obstetrics and Gynecology for 10 days, at the dismission her hemoglobin level was 9.8 $\mathrm{g} / \mathrm{d}$ and she was discharged in a good condition.

The pathology examination showed the chorionic villus penetrated the uterine serosa which confirmed the diagnosis of the placenta percreta at the site of the uterine rupture.

\section{Discussion}

Rupture of the gravid uterus is a life-threatening condition for the fetus and the mother.

This obstetrical complication occurs mainly in the third trimester and is extremely rare during the first and the second trimester.

The major factors in the uterine rupture are the previous caesarean section (scarred uterus) and extended or a obstructed labor; others causes are represented by the grand multiparity ( $>7$ deliveries in obstetric history), advanced maternal age, the use of the uterotonic drugs (like the oxytocin stimulation), abnormal placentation for example placenta previa and/or placenta percreta, macrosomia, obstetrical manipulation, uterine malformations and previous uterine surgery (hysteroscopy, laparoscopic and laparotomic myomectomy) $[7,8]$.

According to the type of hysteroscopic the risks of complications is different: the myomectomy and septum resection had the highest incidence of the complications, while the diagnostic hysteroscopy and polypectomy had the lowest risk $[9,10]$. However medical doctors should inform the patients about the possible complications of hysteroscopy and the people must be informed that the risk is higher immediately after the operation

There are no studies about the correlation between the resistance of the uterus scar and the intensity of uterus contractions, but in any case, ultrasound evaluation of the caesarean uterine scar is essential. Many publications confirm that the characteristic of the good quality of the scar include: scar thickness of $3.5 \mathrm{~mm}$ or more, scar volume verified by $3 \mathrm{D}$ technique up to $10 \mathrm{~cm}$, the homogeneity of the scar, triangular shape of the scar and richer perfusion [4,11]. Histologically the main problem of the uterine scar is collagen deposition because it seems to alter the proliferation, differentiation and the migrations of the basalis layer of the endometrium and the wall of the uterus is weaker.Another important point is the time after myomectomy and it seems that 6 months is the minimum time for the uterus to cicatrize [4].

In the past the loss of uterine tone was the characteristic of the rupture of the uterus, while recent evidence shown that the uterine contraction is not associated to the uterine rupture. The first symptoms of the uterine rupture are not characteristic, so it may be difficult to establish the diagnosis. The first common sign is the fetal bradycardia ( $87 \%$ of the cases) but sudden abdominal pain, vomiting and nausea are also common. Vaginal bleeding, maternal tachycardia or unusual pain during labor are also described [12-14]. In most cases which occur in a hospital setting, timely laparotomy results in safe delivery of the baby and repair of the uterus.

The most common rupture sites were the cornual area and the uterine fundus, but in our case the site is different because the rupture affects the posterior wall. During the pregnancy we see physiologic and anatomic changes and an acute abdomen during the pregnancy often presents atypically and it could be difficult to distinguish a tense abdomen from abnormal pregnancy.

Fetal and maternal mortality is correlated with surgery performed, completeness of rupture, and diagnosis of rupture before rather than after delivery. Hysterectomy (in 33.5\%) was more commonly carried out for rupture of an unscarred than a scarred uterus. Posterior rupture more commonly required hysterectomy than anterior or lateral rupture. The maternal mortality was similar in patients undergoing hysterectomy and those treated by repair of the rupture.

\section{Conclusion}

Uterine rupture is one of the major obstetrical emergencies; the survival of the patient after uterine rupture depends on the time interval between rupture and the intervention and the availability of blood products for transfusion. Rupture of uterus is frequently diagnosed on the basis of altered fetal heart rate pattern, vaginal bleeding, maternal tachycardia or unusual pain during labor. 
The main lesson of this case is that the possibility of a potential uterine rupture should be considered when a gravid woman presents acute abdominal pain, even in a primigravid patient; but in any case, it can remain silent until delivery when it is detected in exploration.

This case report shows the previous uterine surgery should be considered a risk factor for the placenta percreta at the site of the prior scar, which can result in the uterine rupture. Routine sonographic follow-up examinations and careful clinical observations are especially recommended for women with previous intrauterine surgeries.

\section{Authors' contributions}

Debora D'aniello has made substantial contribution to the acquisition and interpration of data, helped to draft and to write the manuscript.

Giusi Santangelo, Valentina Tibaldi and Andrea Giannini helped to the acquisition of data.

Innocenza Palaia and Violante Di Donato have made substantial contribution to the interpretation of data.

Roberto Brunelli, Ludovico Muzii and Pierluigi Bendetti Panici revised the manuscript.

Marco Monti revised the manuscript critically for important intellectual content and approved the version to be submitted.

All authors read and approved the final manuscript.

\section{Declaration of competing interest}

The authors declare that they have no conflicts of interest.

\section{Funding}

This research did not receive any specific grant from funding agencies in the public, commercial, or not-for-profit sectors.

\section{Acknowledgements}

None.

\section{References}

1. Hofmeyr GJ, Say L, Gulmezoglu AM (2005) WHO systematic review of maternal mortality and morbidity: The prevalence of uterine rupture. BJOG 112: 1221-1228.

2. Posthumus L, Donker ME (2017) Uterine rupture in a primigravid patient, an uncommon but severe obstetrical event: a case report. J Med Case Rep 11: 339-342.

3. Chauhan SP, Martin JN, Henrichs CE, Morrison JC, Magann EF (2003) Maternal and perinatal complications with uterine rupture in 142,075 patients who attempted vaginal birth after cesarean delivery: a review of the literature. Am J Obstet Gynecol 189: 408417.

4. Katarzyna MT, Maciej W, Pawel R (2018) Uterine rupture at 28 weeks of gestational after laparoscopic myomectomy-a case report. Menopause Rev 17: 101-104.

5. Kathryn LP, Won R, and Clymer L (2019) Uterine rupture on MRI presenting as nonspecific abdominal pain in a primigravid patient with 28 -week twins resulting in normal neurodevelopmental outcomes at age two. Case Rep Obstet Gynecol 2019: 1-5.

6. Zwart JJ, Richters JM, Ory F, De Vries JIP, Bloemenkamp KWM, et al. (2009) Uterine rupture in the Netherlands: a nationwide population-based cohort study. BJOG 116: 1069-1078.

7. Egbe TO, Halle-Ekane GE, Tchente CN, Nyemb JE, Belley-Priso E (2016) Management of uterine rupture: a case report and review of the literature. BMC Res Notes 9: 492.

8. Topuz S (2004) Spontaneous uterine rupture at an unusual site due to placenta percreta in a 21-week twin pregnancy with previous cesarean section. Clin Exp Obstet Gynecol 31: $239-241$.

9. Zeteroğlu S, Aslan M, Akar B, Ada Bender R, Başbuğ A, et al. (2017) Uterine rupture in pregnancy subsequent to hysteroscopic surgery: A case series. Turk J Obstet Gynecol 14: 252-255.

10. Propst AM, Liberman RF, Harlow BL, Ginsburg ES (2000) Complications of hysteroscopic surgery: predicting patients at risck. Obstet Gynecol 96: 517-520.

11. Basic E, Basic-Cektovic V, Kozaric H, Rama A (2012) Ultrasound evaluation of uterine scar after cesarean section. Acta Inform Med 20: 149-153.

12. Ouzounian JG, Quist-Nelson J, Miller DA, Korst LM (2015) Maternal and fetal signs and symptoms associated with uterine rupture in women with prior cesarean delivery. $J$ Matern Fetal Neonatal Med 28: 1270-1277.

13. Ridgeway JJ, Weyrich DL, Benedetti TJ (2004) Fetal heart rate changes associated with uterine rupture. Obstet Gynecol 103: 506-512.

14. Pakniat H, Soofizadeh N, Khezri MB (2016) Case report Spontaneous uterine rupture after abdominal myomectomy at the gestational age of 20 weeks in pregnancy: A case report. Int J Reprod BioMed 14: 483-486.

Copyright: (C2020 D'aniello D. This is an open-access article distributed under the terms of the Creative Commons Attribution License, which permits unrestricted use, distribution, and reproduction in any medium, provided the original author and source are credited. 